\title{
Time for a culture change? Suboptimal compliance with blood culture standards at a district hospital in Cape Town
}

\author{
M S Abrahams, MB ChB; A C Whitelaw, MB BCh, MSc, FCPath (SA) (Micro); H Orth, MB ChB, MMed (Micro), DTM\&H \\ Division of Medical Microbiology, Faculty of Medicine and Health Sciences, Stellenbosch University, Tygerberg, Cape Town, South Africa, \\ and National Health Laboratory Service, Tygerberg Hospital, Cape Town
}

Corresponding author: M S Abrahams (shareef.abrahams@nhls.ac.za)

\begin{abstract}
Background. The benchmark for contaminated blood cultures (BCs) is $3 \%$. The South African (SA) guideline aims to optimise BC yield and reduce contamination. Data on BC collection practices in SA since the publication of the 2010 SA guideline are lacking.

Objective. To evaluate compliance with the national guideline for the optimal use of $\mathrm{BCs}$ and determine the $\mathrm{BC}$ contamination rate at a local district hospital.

Method. An audit of compliance with 22 BC standards was conducted at a district hospital in Cape Town, SA. Standards were evaluated by reviewing clinical and laboratory data and by a clinician questionnaire.

Results. Of the $425 \mathrm{BCs}$ reviewed, $12.5 \%$ had positive growth, and $4.5 \%$ grew contaminants. Only $33 \%$ of $\mathrm{BC}$ bottles contained the recommended fill volume of $8-10 \mathrm{~mL}$, and $96.9 \%$ of patients had a single BC within a 24 -hour period. Of all the BCs, only $7.8 \%$ had a combined blood volume of at least $20 \mathrm{~mL}$. The yield of pathogens in BCs collected after antibiotic exposure was $4.9 \%$ compared with $7.5 \%$ for those cultures with no prior antibiotic exposure $(p=0.3)$. The overall median needle-to-incubator transport time was 11 hours 25 minutes.

Conclusion. The $\mathrm{BC}$ contamination rate was high and compliance with most standards was variable or not met. The findings may not be generalisable to other hospitals, and we recommend that each institution reviews its own BC practices. Recommendations made to hospital staff included a re-audit following implementation of these recommendations.
\end{abstract}

S Afr Med J 2015;105(12):1039-1043. DOI:10.7196/SAMJ.2015.v105i12.9442



Blood culture $(\mathrm{BC})$ is the 'gold standard' for the detection of micro-organisms in the bloodstream. The 2010 South African (SA) guideline for the optimal use of BCs aims to optimise BC yield and reduce contamination. ${ }^{[1]}$ There are approximately 20000 deaths per day from sepsis worldwide, ${ }^{[2]}$ and $\mathrm{BC}$ plays an integral role in identifying bacteraemia and guiding diagnostic and therapeutic choices. It is also one of the cornerstones of antimicrobial stewardship, which has reduced the overuse of antibiotics and costs in hospitalised patients. ${ }^{[3]}$

The interpretation of $\mathrm{BC}$ results may be complicated by the recovery of potential contaminants. The Clinical and Laboratory Standards Institute (CLSI)'s benchmark for the maximum acceptable percentage of contaminated BCs is $3 \%{ }^{[4]}$ Contaminated BCs are associated with additional laboratory testing, unnecessary prescription of antibiotics and increased patient charges. ${ }^{[5]} \mathrm{A}$ recent audit of BCs among adult patients at GF Jooste Hospital in Cape Town, SA, reported high rates of contamination by coagulase-negative staphylococci (CoNS) ranging from $4.6 \%$ to $9.3 \%$ per year. ${ }^{[6]}$

To our knowledge, there has not been a comprehensive audit of BC collection practices in SA since the publication of the SA guideline. ${ }^{[1]}$

\section{Objectives}

To evaluate compliance with the SA guideline ${ }^{[1]}$ for the optimal collection of BCs and to determine the institutional BC contamination rate at a district hospital in Cape Town.

\section{Methods}

\section{Study design}

We conducted an audit of compliance with 22 BC standards (Table 1) derived from the national guideline. ${ }^{[1]}$ Clinical and laboratory data were extracted over a 3-month period (1 November 2013 - 31 January 2014). All patient data were anonymised. The study was approved by the Research Ethics Committee of Stellenbosch University, Cape Town (Protocol REC S12/09/248).

\section{Setting and patient population}

The audit was conducted at a 282-bed district hospital in Cape Town. The hospital is situated $3.5 \mathrm{~km}$ from the National Health Laboratory Service (NHLS) microbiology laboratory, located at Tygerberg Hospital. A courier service collects specimens from the hospital and delivers them to the laboratory 12 times a day on week days and 8 times a day over weekends. All adult inpatients $(\geq 13$ years) from whom BCs were collected were eligible for inclusion. Mycobacterial BCs were excluded because the mycobacterial BC instrument used was different from that used for routine BCs.

\section{Definitions}

A BC was defined as a sample of blood obtained from a single venepuncture site, irrespective of whether the blood was inoculated into one or multiple BC bottles.

$\mathrm{A} \mathrm{BC}$ was considered to be contaminated if one or more of the following skin flora organisms were identified in only one of a series of BCs: CoNS, Corynebacterium spp., viridans group streptococci, Micrococcus spp., Bacillus spp., and Propionibacterium spp. ${ }^{[7]}$ This included BCs with a potential pathogen, in addition to a contaminant. Polymicrobic BCs with more than one contaminant species were considered as being a single contaminated $\mathrm{BC} \cdot{ }^{[8]}$ If any of the abovementioned skin flora organisms were cultured from a solitary blood culture (SBC) (single BC within a 24-hour period), the medical records and medication charts were retrospectively reviewed. BCs were classified as clinically significant if antibiotic treatment was initiated or 
continued as a therapeutic response to the $\mathrm{BC}$ result, an intravascular line was removed or replaced as a therapeutic response to the $\mathrm{BC}$ result, or the BC result was associated with an expected clinical condition (e.g. graft sepsis, shunt sepsis or endocarditis). All positive BCs with a recognised pathogen were considered to be clinically significant.

Needle-to-incubator transport time was defined as the time difference between $\mathrm{BC}$ collection and insertion of $\mathrm{BC}$ bottles into the $\mathrm{BC}$ instrument. Insertion on the day of sample collection was defined as same-day incubation, and insertion on a different day as delayed incubation.

The institutional $\mathrm{BC}$ contamination rate was defined as the number of contaminated BCs processed during the study period, divided by the total number of BCs performed during that period. ${ }^{[8]}$

\section{Data sources}

- Clinical and laboratory data were extracted from clinical case notes, laboratory request forms, BC bottles, medication charts and the laboratory information system (LIS).

\section{Table 1. Blood culture standards}

S1 BCs should be drawn if there is a clinical suspicion of a bloodstream infection.

S2 Informed consent should be obtained prior to performing a BC.

S3 BCs should be collected prior to administration of antibiotics.

S4 Hand washing should be performed prior to performing a BC.

S5 Hands should be disinfected prior to performing a BC.

S6 Sterile gloves should be used when performing a BC.

S7 BCs should be drawn from peripheral sites.

S8 BCs should be collected from separate venepuncture sites.

S9 Puncture site should be cleaned using appropriate disinfectant.

S10 Skin disinfectant should be allowed time to dry before inserting the needle.

S11 BC bottle tops should be disinfected prior to inoculation.

S12 Bottle-top disinfectant should be allowed to dry prior to inoculation.

S13 Needles should not be exchanged between BC collection and inoculation of $\mathrm{BC}$ bottles.

S14 BC bottles should be inoculated first, if blood is collected for other tests.

S15 Minimum of 2 and maximum of 3 BCs should be drawn within 24 hours

S16 Aerobic BC bottles should be used in a resource-limited setting.

S17 Minimum of $20 \mathrm{~mL}$ of blood ( $10 \mathrm{~mL}$ per bottle) should be obtained for each BC.

S18 BC bottles should be correctly labelled.

S19 Laboratory request form should include patient identifiers, site, date and time of collection, clinical information regarding suspected diagnosis, and contact details of requesting doctor.

S20 BC collection should be documented in the clinical notes

S21 BC bottles should be left at room temperature if there is a delay in transporting them to the laboratory.

S22 BC bottles should be delivered to the laboratory as soon as possible.
- BC bottle volume was determined by subtracting the post-filling weight from the standard pre-filled weight. One $\mathrm{mL}$ of blood weighs approximately $1 \mathrm{~g}$. The manufacturer (bioMérieux) recommends that a sample volume of $8-10 \mathrm{~mL}$ blood be provided per bottle and that the maximum sample volume should not exceed $10 \mathrm{~mL}$ per bottle.

- Needle-to-incubator transport time was calculated using the date and time of $\mathrm{BC}$ collection documented on the laboratory request form and the times available on the LIS.

- A clinician questionnaire was designed to evaluate standards relating to obtaining informed consent, $\mathrm{BC}$ ordering practices, skin and bottletop antisepsis, $\mathrm{BC}$ collection technique, and storage of $\mathrm{BC}$ bottles.

\section{Data evaluation (standards numbered as per Table 1)}

- Standard 1 was evaluated by reviewing the working diagnosis in the clinical case notes at the time of BC collection.

- Standards 2, 4-6,8 - 14 and 21 were evaluated by a clinician questionnaire. Selected clinician responses were ranked as follows: always (>95\%), usually (50 - 95\%), sometimes (5 - 50\%), rarely $(<5 \%)$, and uncertain.

- Standard 3 was evaluated by reviewing laboratory request forms, medication charts and the clinician questionnaire.

- Standard 7 was evaluated by reviewing the site of BC collection as indicated on the laboratory request forms.

- Standards 15 - 20 and 22 were evaluated by reviewing laboratory request forms, $\mathrm{BC}$ bottles, clinical case notes and the LIS.

\section{Laboratory procedures}

BC bottles were transported to the laboratory and incubated either until they flagged positive, or for 5 days (or longer if extended incubation was required) in the BacT/Alert (bioMérieux, SA) BC system. Broths from positive bottles were Gram-stained and further identified using standard techniques. ${ }^{[9]}$

\section{Statistical analysis}

Conventional descriptive methods were used for data analysis Percentages were reported for categorical variables and median and interquartile ranges (IQRs) for continuous variables. Selected

Table 2. Micro-organisms recovered from blood cultures*

\begin{tabular}{ll}
\hline & Isolates, $\boldsymbol{n}$ \\
\hline Pathogens & 13 \\
Enterobacteriaceae & 8 \\
Staphylococcus aureus & 6 \\
Streptococcus pneumoniae & 5 \\
Pseudomonas aeruginosa & 2 \\
Cryptococcus neoformans & 2 \\
Staphylococcus hominis ${ }^{\dagger}$ & 1 \\
Erysipelothrix rhusiopathiae & 1 \\
Streptococcus anginosus & \\
Contaminants & 11 \\
Coagulase-negative Staphylococcus spp. & 4 \\
Bacillus spp. & 3 \\
Viridans group streptococci & 1 \\
Corynebacterium spp. & 1 \\
Micrococcus spp. & \\
*Total of 425 blood cultures with 58 isolates. \\
'solate was cultured from two separate blood cultures in a patient with vascular graft sepsis.
\end{tabular}


categorical data were evaluated using the $\chi^{2}$ test, and a $p$-value of $<0.05$ was regarded as statistically significant.

\section{Results}

We reviewed $425 \mathrm{BCs}$ (531 BC bottles) collected from 353 patients in different wards over a 3 -month period. Table 2 shows the organisms recovered from BCs. More than one bacterial species was demonstrated in $1.2 \%(5 / 425)$ of BCs. The BC contamination rate was $4.5 \%(19 / 425)$ (Table 3).

The clinician questionnaire response rate was $70.0 \%$ (30/43). Compliance with standards relating to obtaining informed consent, skin and bottle-top antisepsis and BC collection technique was variable (Fig. 1). Forty-eight percent (13/27) of doctors reported using only chlorhexidine for skin antisepsis; $26.0 \%$ (7/27) reported using chlorhexidine and 70\% alcohol; 18.5\% (5/27) reported using only $70 \%$ alcohol; $3.7 \%(1 / 27)$ reported using both chlorhexidine and povidone; and $3.7 \%(1 / 27)$ reported that they did not know which skin disinfectant to use.

The indications for performing BCs were diverse, with sepsis and fever being the most common indications (Table 4).

We were able to determine the temporal relationship between BC collection and antibiotic exposure for $81.7 \%$ (343/425) of

Table 3. Total number of, and proportion of, blood culture contaminants expressed in terms of number of blood cultures and number of blood culture bottles

\begin{tabular}{|c|c|c|}
\hline & $\begin{array}{l}\text { Blood cultures } \\
(N=425) \\
n(\%)\end{array}$ & $\begin{array}{l}\text { Blood culture } \\
\text { bottles }(N=531) \\
n(\%)\end{array}$ \\
\hline Positive blood cultures & $53(12.5)$ & $64(12.1)$ \\
\hline Significant positives & $34(8.0)$ & $43(8.1)$ \\
\hline Contaminants & $19(4.5)$ & $21(4.0)$ \\
\hline
\end{tabular}

BCs. Of these cultures, 53.6\% (184/343) were collected after in-hospital exposure to antibiotics in the preceding 24 hours. The overall yield of BCs collected after in-hospital antibiotic exposure was $7.6 \%(14 / 184)$ and that of those collected with no prior in-hospital antibiotic exposure was $15.1 \%(24 / 159)$ (odds ratio (OR) 0.46 ; $95 \%$ confidence interval (CI) $0.23-0.93 ; p=0.04$ ). However, the yield of pathogens in BCs collected after antibiotic exposure was $4.9 \%(9 / 184)$ compared with $7.5 \%(12 / 159)$ for those cultures with no prior antibiotic exposure (OR 0.63 ; 95\% CI 0.26 - 1.54; $p=0.3$ ).

The site of BC collection was indicated on $0.7 \%(3 / 425)$ of laboratory request forms. Only one request form indicated that the $\mathrm{BC}$ was drawn from a central venous catheter (CVC).

\section{Table 4. Indications for blood culture}

\begin{tabular}{|c|c|c|}
\hline Indication & Frequency & $\%$ \\
\hline Sepsis & 110 & 25.9 \\
\hline Fever & 78 & 18.4 \\
\hline Pneumonia & 50 & 11.8 \\
\hline Urinary tract infection & 15 & 3.5 \\
\hline Skin and soft-tissue infection & 14 & 3.3 \\
\hline Meningitis & 12 & 2.8 \\
\hline Diarrhoea & 8 & 1.9 \\
\hline Delirium & 6 & 1.4 \\
\hline Endocarditis & 6 & 1.4 \\
\hline Unclear $^{\dagger}$ & 70 & 16.5 \\
\hline Miscellaneous & 55 & 12.9 \\
\hline
\end{tabular}

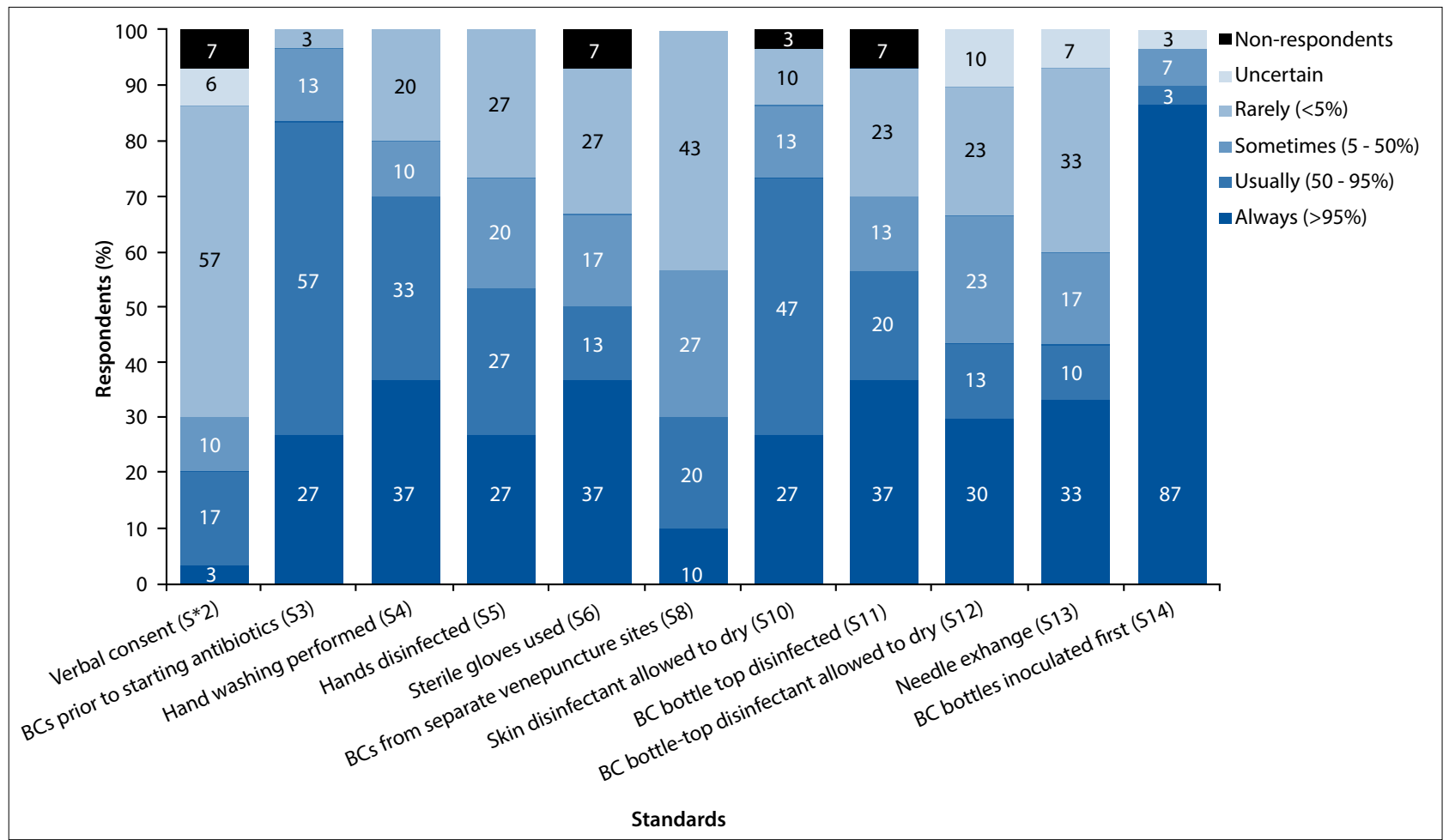

Fig. 1. Standards evaluated and ranked by clinician questionnaire. $\left({ }^{\star} S=\right.$ standard.) 
Seventy-five percent (318/425) of BCs comprised a single BC bottle, $24.7 \%(105 / 425)$ two bottles, and $0.2 \%(1 / 425)$ three bottles. The majority $(96.9 \%, 342 / 353)$ of patients had a single BC in a 24 -hour period. Only $3.1 \%(11 / 353)$ of patients had two BCs and no patients had three or more BCs within a 24-hour period. Eighty-six percent (457/531) of all BC bottles received were aerobic bottles. Thirtythree percent $(175 / 531)$ of $\mathrm{BC}$ bottles contained a fill volume of 8 - $10 \mathrm{~mL}, 46.0 \%(245 / 531)$ contained $<8 \mathrm{~mL}$, and $21.0 \%(111 / 531)$ contained $>10 \mathrm{~mL}$. Only $7.8 \%(33 / 425)$ of BCs collected had a blood volume of at least $20 \mathrm{~mL}$. Although the pathogen yield from BC bottles with a volume of $<8 \mathrm{~mL}(12 / 245,4.9 \%)$ was lower than the yield from BC bottles with the recommended volume (14/175, $8.0 \%$ ), this difference was not statistically significant (OR $0.59 ; 95 \%$ CI $0.26-1.31 ; p=0.19)$.

We were unable to trace the clinical folders or clinical entry sheets for $7.3 \%(31 / 425)$ of BCs. BC collection was documented in the clinical folders for $85.5 \%$ (337/394) of BCs. Ninety-three percent (395/425) of the request forms contained patient stickers with the relevant patient details. The remainder of the forms comprised handwritten patient identifiers, all of which contained a minimum of three data sets (first name, surname and folder number). All laboratory request forms indicated the date of $\mathrm{BC}$ collection, but only $76.2 \%(324 / 425)$ indicated both the date and time. Of all the request forms reviewed, $72.5 \%$ (308/425) contained clinical information. The name of the requesting doctor was provided on $98.4 \%$ (418/425) of forms, but only $1.2 \%$ (5/425) of forms contained clinician contact details. All BC bottles were correctly labelled.

Seventy-seven percent (23/30) of clinicians reported leaving BCs at room temperature if there was a delay in transporting them to the laboratory. However, $23 \%$ (7/30) failed to respond to this question. The overall median needle-to-incubator transport time was 11 hours 25 minutes (IQR 7 hours 6 minutes - 16 hours 53 minutes). For sameday incubation the median needle-to-incubator transport time was 7 hours 15 minutes (IQR 5 hours 30 minutes - 9 hours 28 minutes), and for delayed incubation it was 16 hours 24 minutes (IQR 12 hours 23 minutes - 22 hours 30 minutes).

Sixty-three percent (19/30) of clinicians indicated that they were unaware of the SA guideline ${ }^{[1]}$ and $70.0 \%(21 / 30)$ were unaware of the local NHLS specimen collection manual.

\section{Discussion and conclusion}

The main findings of our audit related to poor compliance with hand hygiene practices, lack of use of sterile gloves and inadequate skin and bottle-top antisepsis. This was clearly reflected in the high BC contamination rate of $4.5 \%$.

Controversy still exists as to whether sterile gloving should routinely be used during $\mathrm{BC}$ collection. However, a randomised cross-over trial by Kim et al. ${ }^{[10]}$ found that routine use of sterile gloves was associated with a reduction in $\mathrm{BC}$ contamination.

The SA guideline refers to the use of povidone or alcohol solution to disinfect the puncture site. ${ }^{[1]}$ However, a recent systematic review and meta-analysis has concluded that alcoholic chlorhexidine solutions showed a statistically significant reduction in BC false positives compared with aqueous povidone-iodine. ${ }^{[11]}$ Given the current available data, we strongly recommend the use of alcoholic chlorhexidine for skin disinfection.

The method of applying the skin antiseptic is also important. Traditionally, the concentric circle method was required if aqueous iodophors were used after the alcohol step to prevent reintroduction of contaminants to previously cleansed areas. There are no data supporting applying alcoholic disinfectants in an outward concentric circle, but vigorous friction is important. ${ }^{[12]}$ If palpation of the vein is necessary after skin disinfection, the gloved finger should be cleansed with the antiseptic agent and allowed to dry before touching the site.

$\mathrm{BC}$ bottle tops are not necessarily sterile, even though they are covered with a lid ${ }^{[13]}$ Cleaning the $\mathrm{BC}$ bottle tops with antiseptic prior to specimen inoculation decreases contamination. ${ }^{[8]}$

Other pre-analytical factors that may also have played a role in the high $\mathrm{BC}$ contamination rate but were not explored in our study include whether or not the venepuncture site was repalpated after skin antisepsis, patient-related factors (e.g. unco-operative patients or difficult anatomy for venepuncture), and ward factors (e.g. high staff turnover, inexperienced staff, BC equipment lacking or difficult to locate).

Other significant findings of our audit relate to prior administration of antibiotics, high number of SBCs, inadequate blood volume and prolonged needle-to-incubator transport time. All these factors are likely to contribute to the low BC yield of true pathogens found in this study.

Recent antibiotic treatment can have a significant impact on the $\mathrm{BC}$ results by decreasing the sensitivity of the test. $\mathrm{BC}$ collection before initiation of antimicrobial therapy is recommended as a standard of care in international sepsis guidelines. ${ }^{[14]}$

The number of SBCs was unacceptably high. The SA guideline ${ }^{[1]}$ recommends two or more BCs in cases where sepsis is suspected; this is supported by the CLSI and the recent international guidelines of the Surviving Sepsis Campaign. ${ }^{[4,14]}$ Two previous Q-Probes studies in the USA evaluated SBC collections as a pre-analytic quality indicator of BC practice. ${ }^{[15]}$ Of $289572 \mathrm{BCs}$, the median proportion of SBCs per institution was $10.1 \%$. These studies found that one of the most common reasons for not performing a second culture in adults was that the doctor believed that one culture was sufficient. However, we did not explore the reasons for the high rate of SBCs, but it is probably due to a variety of factors including lack of awareness, time pressures, lack of BC equipment and difficult anatomy for venepuncture.

A striking feature of our audit was that the vast majority of $\mathrm{BC}$ bottles were under-filled. The volume of blood tested is the single most important variable for the detection of bacteria and yeast from BCs. ${ }^{[16]}$ Cockerill et al ${ }^{[17]}$ demonstrated that yields from $20 \mathrm{~mL}$ and $30 \mathrm{~mL}$ of blood were $29.8 \%$ and $47.2 \%$ greater, respectively, than those from $10 \mathrm{~mL}$ of blood. Most bacteraemias in adults have a low density of micro-organisms, and $20-30 \mathrm{ml}$ per $\mathrm{BC}$ is therefore recommended. ${ }^{[4]}$ Of all the BC bottles, $21.0 \%$ were overfilled. While underfilling of $\mathrm{BC}$ bottles reduces the sensitivity, overfilling of bottles may cause the $\mathrm{BC}$ system to flag positive falsely.

BCs received at the Tygerberg NHLS laboratory are not inserted into the continuous-monitoring $\mathrm{BC}$ instrument on a 24-hour basis; those received after $22 \mathrm{~h} 00$ are not pre-incubated, but stored at room temperature until the next day. The extended delay between BC collection and incubation is not recommended, as this may delay or impede the detection of growth by the $\mathrm{BC}$ instrument. ${ }^{[18]}$ However, the actual time at which a reduction in $\mathrm{BC}$ sensitivity occurs because of delays in loading $\mathrm{BC}$ bottles is not clearly established. According to the UK Standards for Microbiology Investigations, BC bottles should be incubated as soon as possible, and within a maximum of 4 hours. ${ }^{[19]}$ The strengths of our clinical audit were:

- We incorporated both clinical and laboratory data and it was not solely laboratory based.

- We attempted to evaluate actual practice and not only the BC contamination rate.

- We incorporated all probable skin contaminants and not only CoNS when we calculated the $\mathrm{BC}$ contamination rate.

- We used the number of BCs (and not the number of BC bottles) to calculate the $\mathrm{BC}$ contamination rate. 
- When we cultured a probable contaminant from an SBC, we did not use only the identification of the organism, but also clinical parameters, before making a judgement about the clinical significance of the isolate.

The limitations of our audit were:

- We had limited quantitative data on some of the pre-analytic procedures, but they nevertheless provided insight into BC collection practices.

- We inferred the indication for BCs from the working clinical diagnosis documented in the clinical case notes at the time of $\mathrm{BC}$ collection, but we did not evaluate specific clinical parameters or results from other special investigations.

- When more than one BC bottle was submitted for the same patient, using the same laboratory request form, it was counted as one $\mathrm{BC}$ (unless otherwise specified); this may have led to an underestimation of the number of BCs.

- When the site of BC collection was not indicated, it was assumed that it was collected from a peripheral site, which may have led to an underestimation of the number of BCs collected from CVCs.

- We did not audit the availability of equipment (phlebotomy materials, BC bottles and sterile BC packs) for performing BCs.

In conclusion, our audit showed a high BC contamination rate. It further demonstrated that compliance with most standards at the hospital was variable or not met. However, our findings may not be generalised to other hospitals and we therefore recommend that each institution reviews its own $\mathrm{BC}$ practices.

\section{Recommendations for improvement}

The audit report was presented to the hospital staff with the following recommendations:

- Staff be provided with electronic copies of the SA guideline ${ }^{[1]}$ and the NHLS specimen collection manual.

- Pictorial wall charts be posted in each ward, outlining best-practice recommendations.

- $\mathrm{BC}$ record stickers be used, indicating the name of the patient and doctor, and the date, time, site and indication of BC.

- Weekly antibiotic stewardship rounds be used as a platform to educate clinicians regarding best practice when collecting BCs.

- Pre-assembled BC collection packs with procedural checklists be used.

- Winged blood collection sets (needle and adaptor) be used.

- A revised laboratory request form be used, with a designated space for clinicians to clearly indicate the BC collection.

- Alcoholic chlorhexidine be used as a skin disinfectant in adults.

- Laboratory reports be accompanied by a remark recommending the number of BCs and volume of blood, per bottle, for adult patients.
- Pre-analytical factors associated with prolonged needle-to-incubator transport time be investigated.

- BCs be loaded on the BC instrument on a 24-hour basis.

- The audit be repeated after implementation of interventions.

In addition, the regular use of quality indicators (such as blood volume, contamination rate, $\mathrm{SBC}$ rate, positivity rate, number of $\mathrm{BCs}$ requested per 1000 patient-days, needle-to-incubator transport time and number of rejected samples) with monitoring and feedback may strengthen the above interventions.

Acknowledgements. We thank staff at the study hospital and the Tygerberg NHLS laboratory. We gratefully acknowledge the assistance of Sr Marietjie Bester, Kristien Nel, and Jeremy Goodway with the data collection and analysis.

\section{References}

1. Ntusi N, Aubin L, Oliver S, et al. Guideline for the optimal use of blood cultures. S Afr Med I 2010;100(12):839-843.

2. Daniels R. Surviving the first hours in sepsis: Getting the basics right (an intensivist's perspective). J Antimicrob Chemother 2011;66(Suppl2):ii11-ii23. [http://dx.doi.org/10.1093/jac/dkq515]

3. Katsios CM, Burry L, Nelson S, et al. An antimicrobial stewardship program improves antimicrobial treatment by culture site and the quality of antimicrobial prescribing in critically ill patients. Crit Care 2012;16:R216. [http://dx.doi.org/10.1186/cc11854]

4. Clinical Laboratory Standards Institute (CLSI). Principles and Procedures for Blood Cultures; Approved Guideline. CLSI Document M47-A. Wayne, PA: CLSI, 2007.

5. Bates DW, Goldman L, Lee TH. Contaminant blood cultures and resource utilization. The true consequences of false-positive results. JAMA 1991;265(3):365-369. [http://dx.doi.org/10.1001/jama.265.3.365]

6. Kenyon CR, Fatti G, Schrueder N, et al. The value of blood culture audits at peripheral hospitals. S Afr Med I 2012;102(4):232-233.

7. Bekeris LG, Tworek JA, Walsh MK, et al. Trends in blood culture contamination: A College of American Pathologists Q-Tracks study of 356 institutions. Arch Pathol Lab Med 2005;129(10):1222-1225.

8. Schifman RB, Strand CL, Meier FA, et al. Blood culture contamination: A College of American 8. Schifman RB, Strand CL, Meier FA, et al. Blood culture contamination: A College of American
Pathologists Q-Probes study involving 640 institutions and 497134 specimens from adult patients. Pathologists Q-Probes study involving 640
Arch Pathol Lab Med 1998;122(3):216-221.

Arch Pathol Lab Med 1998;122(3):216-221.
9. Forbes BA, Sahm DF, Weisfeld AS. Bloodstream infections. In: Bailey and Scott's Diagnostic 9. Forbes BA, Sahm DF, Weisfeld AS. Bloodstream infect
Microbiology. 11th ed. St Louis, MO: Mosby, 2007:778-797.

10. Kim NH, Kim M, Lee S, et al. Effect of routine sterile gloving on contamination rates in blood culture: A cluster randomized trial. Ann Intern Med 2011;154(3):145-151. [http://dx.doi.org/10.7326/00034819-154-3-201102010-00003

11. Caldeira D, David C, Sampaio C. Skin antiseptics in venous puncture-site disinfection for prevention of blood culture contamination: Systematic review with meta-analysis. J Hosp Infect 2011;77(3):223232. [http://dx.doi.org/10.1016/.j.hin.2010.10.015]

12. Baron EJ, Weinstein MP, Dunne WM Jr, Yagupsky P, Welch DF, Wilson DM. Cumitech 1C, Blood Cultures IV. Washington, DC: ASM Press, 2005.

3. Hall KK, Lyman JA. Updated review of blood culture contamination. Clin Microbiol Rev 2006:19(4):788-802. [http://dx.doi.org/10.1128/CMR.00062-05]

14. Dellinger RP, Levy MM, Rhodes A, et al. Surviving sepsis campaign: International guidelines for Dellinger RP, Levy MM, Rhodes A, et al. Surviving sepsis campaign: International guidelines for
management of severe sepsis and septic shock: 2012. Crit Care Med 2013;41(2):580-637. [http://dx.doi. rg/10.1097/CCM.0b013e31827e83af

15. Schiffman RB, Bachner P, Howanitz PJ. Blood culture quality improvement: A College of American Pathologists Q-Probes study involving 909 institutions and 289572 blood culture sets. Arch Pathol Lab Med 1996;120:999-1002

16. Weinstein MP. Current blood culture methods and systems: Clinical concepts, technology, and interpretation of results. Clin Infect Dis 1996;23(1):40-46. [http://dx.doi.org/10.1093/clinids/23.1.40]

17. Cockerill FR 3rd, Wilson JW, Vetter EA, et al. Optimal testing parameters for blood cultures. Clin Infect Dis 2004;38(12):1724-1730. [http://dx.doi.org/10.1086/421087]

18. Sautter RL, Bills AR, Lang DL, Ruschell G, Heiter BJ, Bourbeau PP. Effects of delayed-entry conditions on the recovery and detection of microorganisms from BacT/ALERT and BACTEC blood culture bottles. J Clin Microbiol 2006:44(4):1245-1249. [http://dx doi ro/10.1128/JCM.44.4.1245-1249.2006]

19. Investig Microbiology Investigations. B 37 Issue 7. http://www.hpa.org.uk/SMI/pdf (accessed 12 September 2013).

Accepted 13 October 2015 